\title{
1 Effect of Nitrogen Doping in the Few Layer Graphene Cathode of an Aluminum Ion Battery
}

2 Anthony Childress, ${ }^{1}$ Prakash Parajuli, ${ }^{1}$ Samuel Eyley, ${ }^{2}$ Wim Thielemans, ${ }^{2}$ Ramakrishna Podila, ${ }^{1,}$ 3 Apparao M. Rao ${ }^{1, *}$

41 Clemson Nanomaterials Institute and Department of Physics and Astronomy, Clemson 5 University, Clemson, SC 29625 USA.

62 Renewable Materials and Nanotechnology Research group, Department of Chemical 7 Engineering, KU Leuven, Campus Kulak Kortrijk, Etienne Sabbelaan 53 box 7659, 8500 Kortrijk, 8 Belgium.

$9 \quad *$ Corresponding author: arao@g.clemson.edu

10 Abstract:

11 We examine the effect of nitrogen dopants in Few-layer graphene (FLG) cathodes on anion 12 intercalation. Different nitrogen dopant configurations within the FLG were achieved by varying 13 the synthesis parameters and their effects are compared. The dopant configurations of the samples 14 were determined via X-ray photoelectron spectroscopy (XPS) and formation energies were 15 calculated using density functional theory, allowing correlation between cell performance and 16 dopant configuration. The reduced ion intercalation within the nitrogen doped FLG, and thus poor 17 charge/discharge characteristics of nitrogen doped FLG cathodes, is attributed to the reduced 18 mobility of the chloroaluminate ions in the presence of nitrogen dopants.

20 The use of few-layer graphene (FLG) as a cathode in aluminum-ion batteries (AIBs) has recently 21 attracted much interest, largely due to the 2015 report by Lin et al.[1] AIBs have the potential to 
1 provide a less costly alternative to lithium based cells and provide several benefits: aluminum is

2 highly abundant compared to lithium, it possesses a large gravimetric $\left(2980 \mathrm{~mA} \mathrm{~h} \mathrm{~g}^{-1}\right)$ and

3 volumetric $\left(8063 \mathrm{~mA} \mathrm{~h} \mathrm{~g}^{-1}\right)$ capacity, and AIBs use non-flammable ionic liquids as electrolytes.

4 The use of FLG as a cathode also allows for greater power density than that of LIBs. Past efforts

5 at creating a rechargeable AIB were hampered by the difficulties of finding a suitable cathode

6 material. Graphite is typically used as an energy storage medium via intercalation of ions, but bulk

7 graphite suffers from disintegration, low discharge voltage, and poor cycle life in AIBs that rely

8 on large chloroaluminate ions. FLG has a much more flexible structure than bulk graphite, and it

9 was this property that was leveraged by Lin et al. to create a viable AIB that allowed facile ion 10 intercalation. Since then, many other reports demonstrating improved performance of FLG-based 11 AIBs have appeared in the literature.[2-7] We showed in a previous study that AIBs exhibit the 12 best performance when the FLG is fully intercalated to stage I by the chloroaluminate ions.[8] Liu 13 et al. showed that "frizzy" carbon nanoscrolls made from FLG exhibit rapid electronic 14 transportation channels, superior anion storage capability, and outstanding ability to accommodate 15 a large volume expansion during the cycling process in AIBs due to edge defects present in the 16 cathode.[9] Similarly, following our work on defect-engineered FLG supercapacitors, Yu et al. 17 showed that AIBs with plasma etched graphene exhibit high capacity, and excellent cycling 18 ability.[10]

19 It is well-known that the introduction of dopants such as nitrogen improves the performance of 20 nanocarbon electrodes in many energy storage applications. For example, nitrogen doped FLG 21 (N-FLG) was found to improve the capacity and rate capability of potassium-ion cells when used 22 as an anode by providing reactive sites that are amenable to charge transfer.[11] Nitrogen-doped 23 carbon nanofibers derived from polypyrrole were found to improve the function of sodium-ion 
2 Polypyrrole-functionalized graphene sheets have also been used to improve sodium-ion batteries

3 in the same manner.[13] Lithium-ion cells have benefitted from N-doped graphitic electrodes; one

4 to two-layer N-doped graphene showed almost double the capacity of pristine analogs due to

5 increased surface defects,[14] silicon nanowires coated with N-doped graphene improved lithium

6 adsorption through the presence of dangling bonds,[15] and N-doped graphite oxide sheets allow

7 greater lithium-ion capacity.[16] In all of the above [10-15], the intercalating species are

8 monatomic cations which can benefit from the increased electronegativity provided by nitrogen

9 dopant atoms within the carbon lattice. Due to the benefits of nitrogen dopants that have been

10 observed in other battery chemistries (K, Na, and Li-ion), here we investigate the role of such 11 dopants in AIBs.

12 Nitrogen dopants are known to adopt three main configurations within the graphene lattice, namely 13 graphitic, pyridinic, and pyrrolic (Fig. 1a). Each configuration alters the local electronic density of 14 states around the nitrogen site in the graphene lattice in a specific manner.[17,18] The pyrrolic 15 configuration exhibits the greatest local electronic density of states at the Fermi level $\left(E_{\mathrm{F}}\right)$ and is 16 most reactive among the three configurations due to the presence of pentagons in the graphene 17 lattice at the dopant site. Given that pyridinic and pyrrolic configurations induce atomic vacancies 18 that act as electron acceptors (unlike electron donating $\mathrm{N}$-dopants), the shift in the $E_{\mathrm{F}}$ of graphene 19 greatly depends upon N-dopant configuration. Nitrogen in the graphitic configuration results in an 20 upshift of $E_{\mathrm{F}}$ or $n$-doping while pyridinic results in a downshift of $E_{\mathrm{F}}$ or $p$-doping due to the 21 dangling bonds of the carbon vacancies.[19,20] In our previous work, we found that FLG with 22 different ratios of the three nitrogen configurations can be produced by altering the ratio of nitrogen 23 containing species introduced during the chemical vapor deposition (CVD) process.[21] Herein, 
1 we use the CVD growth method in addition to post-growth treatment of FLG to obtain N-FLG

2 cathodes with varying concentrations and configurations of nitrogen.

3 A detailed synthesis of the FLG cathode can be found in Ref. [8]. Briefly, three different N-FLG

4 samples were prepared; two via in-situ doping during CVD growth and one via post-growth

5 treatment. For the samples prepared via CVD, Ar gas was bubbled at $50 \mathrm{sccm}$ for the entire 60

6 minutes growth period through acetonitrile (sample \#1) or a 1:3 volume mixture of

7 acetonitrile:benzylamine $\left(\mathrm{ACN}: \mathrm{BzNH}_{3}\right)$ (sample \#2). For post-growth processing, pristine FLG

8 samples were first exposed to 2 minutes of $100 \mathrm{~W} \mathrm{~N}_{2}$ plasma followed by annealing in ACN vapors

9 at $600{ }^{\circ} \mathrm{C}$ for one hour (sample \#3). Their respective Raman spectra are shown in Fig. 1b, and have 10 been normalized to the prominent $G$-band found at $\sim 1585 \mathrm{~cm}^{-1}$. The magnitude of the disorder or $11 D$-band present at $\sim 1350 \mathrm{~cm}^{-1}$ is related to the amount of defects present in the graphene lattice.[22]

12 As evident in Fig. 1b, the pristine samples exhibit a very weak $D$-band while all N-FLG samples 13 show a relatively strong $D$-band. Notably, sample \#3 exhibited the smallest $D$-band intensity, 14 which is attributed to the post-synthesis annealing of defects within the graphene lattice. As shown 15 in Figs. 1c-e, we ascertained the nitrogen content and doping configuration via XPS, the details of 16 which can be found in the supporting information. In the case of samples \#1 and \#2, the nitrogen 17 content was found to be $0.30 \%$ and $0.13 \%$ respectively, where the $\mathrm{N}$-dopants are mainly present 18 in the graphitic configuration. The post-plasma annealing of sample \#3 increased the nitrogen 19 content to $1.74 \%$ with predominantly pyridinic type dopants.

20 The electrochemical properties of all samples were analyzed by assembling transparent pouch 21 cells, which allowed for simultaneous Raman characterization during charge/discharge cycling 22 and cyclic voltammetry. The Raman spectra of pristine FLG and N-FLG during the charging 23 process can be seen in Fig. 2a. The spectra in the left panel correspond to the pristine FLG cathode 
1 while the spectra in the right panel correspond to samples \#1 and \#2, which were very similar.

2 Sample \#3, which showed the largest concentration of nitrogen defects, displayed very poor

3 charging characteristics likely due to a lack of intercalation. A cyclic voltammogram of sample \#3

4 is shown in Fig. S1. In the Raman spectra shown in Fig. 2a, the bottom most spectrum in each

5 series was collected prior to charging. In this initial state, as expected, the $G$-band is found at

$6 \sim 1584 \mathrm{~cm}^{-1}$. While the intercalation commences at $\sim 1.9 \mathrm{~V}$ for the pristine FLG, it was found to

7 occur at $2.10 \mathrm{~V}$ for samples \#1 and \#2. As the voltage further increases, the $G$-band splits due to

8 staging of the $\mathrm{AlCl}_{4}{ }^{-}$within the $\mathrm{FLG}$ layers, forming a graphite intercalation compound.[23-25]

9 For the pristine FLG there is an additional splitting of the $1584 \mathrm{~cm}^{-1}$ Raman peak at higher voltage 10 as the FLG transitions to a stage I intercalation compound.[8] The staging process should also 11 result in a complete suppression of the original $1584 \mathrm{~cm}^{-1}$ peak, which is still present in the spectra 12 of samples \#1 and \#2. These results indicate that the N-FLG samples prepared via in-situ CVD 13 doping (viz., samples \#1 and \#2) do not charge properly due to poor intercalation, preventing the 14 FLG from even reaching a stage II intercalation compound. This is also true for sample \#3, where 15 charging of the cell could not be accomplished due to failure of the cell before the maximum 16 voltage of $2.35 \mathrm{~V}$ could be reached. The transition between stages of a graphite intercalation 17 compound can also be seen in the cyclic voltammograms, where a peak in the current marks the 18 transition from one stage to the next. For example, the cyclic voltammograms for samples \#1 and $19 \# 2$ are shown in Fig. 2b, where both samples are represented by one curve. The current peak at the 20 lowest voltage delineates the beginning of intercalation associated with a dilute stage I compound. 21 The subsequent peaks mark the transition to stage II and finally stage I for the peak at the highest 22 voltage. For samples $\# 1$ and $\# 2$, the current peaks associated with intercalation are very broad to 23 the point where the two neighboring peaks are almost indistinguishable unlike the pristine FLG 
1 cathode. In cyclic voltammetry, the applied voltage drives the charge-transfer process and

2 intercalation. In the case of samples \#1 and \#2, the initial intercalation proceeds relatively slowly

3 as evidenced by the broad peaks. This is in contrast to the profile of the pristine FLG that shows

4 three easily distinguishable peaks, where the third peak beginning to form at the voltage of $\sim 2.3 \mathrm{~V}$

5 is associated with a transition to a stage I intercalation compound. Charging of samples \#1, \#2, and

6 \#3 also resulted in the evolution of chlorine gas, which was inferred from the expansion of the

7 pouch cells during cycling. The chlorine gas would evolve from the oxidation of the $\mathrm{AlCl}_{4}{ }^{-}$ions at

8 the higher end of the voltage range as known from the cell chemistry. Such gas formation is also

9 consistent with the well-known catalytic properties of N-doped graphene.[26-29] Clearly, the

10 presence of nitrogen dopant atoms within the carbon lattice adversely influenced the intercalation

11 of chloroaluminate ions, which is in contrast to monatomic species which benefit from the presence

12 of nitrogen dopants.

13 Modeling of the charge transfer between the graphene planes and $\mathrm{AlCl}_{4}{ }^{-}$ions was carried out via 14 density functional theory (DFT) using Quantum ESPRESSO software to gain insights into the 15 interaction of the $\mathrm{AlCl}_{4}{ }^{-}$and the nitrogen dopants. The parameters used in the calculations can be 16 found in our previous publication.[8] Modelling was carried out for pristine FLG and different 17 nitrogen configurations in the graphene lattice. Figures $3 \mathrm{a}$ and $\mathrm{b}$ represent respectively the charge 18 transfer model for the chloroaluminate ion in pristine and pyridinic graphene. The red color 19 indicates electron extraction from the graphene planes by the ion, the magnitude of which was 20 found by integration of the shaded regions in Fig. 3c. The estimated amount of charge transfer is $21 \sim 0.8$ electrons per intercalant ion, which remains roughly constant even with the presence of 22 nitrogen. The different dopant configurations give rise to a similar amount of charge transfer with 23 respect to the projection of charge transfer along z-axis. (i.e. perpendicular to graphene plane). 
1 However, the spatial distribution of charge transfer from the planes parallel to graphene is different

2 for each doping configuration with the same isosurface value of charge transfer. The graphitic

3 configuration is shown in Fig. S2.

4 For modeling the various nitrogen dopant configurations, the $\mathrm{AlCl}_{4}{ }^{-}$ion and the adjacent graphene 5 planes were first allowed to relax individually before they were brought together as the intercalated 6 system and allowed to relax once again. The site for the intercalant was chosen as one of many 7 possible configurations in order to address computational cost. The difference of energy values 8 between the individual and intercalated systems was then calculated as the formation energy. Thus, 9 a positive energy value indicates that intercalation is more favorable. From this standpoint, the 10 pristine FLG and pyrrolic/pyridinic hybrid systems are found to be relatively more favorable than 11 the pure pyridinic analog with formation energies of $0.998 \mathrm{eV}, 0.938 \mathrm{eV}$ and $0.896 \mathrm{eV}$ respectively. 12 Moreover, in the graphitic configuration, the $\mathrm{AlCl}_{4}^{-}$ion was most strongly bound between 13 graphene layers with a binding energy of $\sim 2.23 \mathrm{eV}$ per ion. It is well known that intercalation 14 depends not only on the formation energy but also on the energy landscape of the gallery.[30] In 15 the case of graphitic defects, the $\mathrm{AlCl}_{4}^{-}$ions are trapped in a relatively deep energy well (of the 16 17 the mobility of ions in the gallery. Thus, the reduced mobility of $\mathrm{AlCl}_{4}{ }^{-}$ions in the presence of 18 graphitic defects is proposed as the cause of poor intercalation in samples \#1, \#2. This result is 19 consistent with our experimental in situ Raman spectroscopy that showed a lack of stage I 20 intercalation and breakdown of electrolyte for samples \#1 and \#2 whose defects are predominantly 21 of the graphitic type as inferred from the XPS data. Sample \#3 which had the greatest amount of 22 nitrogen also showed the worst performance. Based on the area under the curves of the XPS 23 spectra, the graphitic type dopants comprise approximately $10 \%$ of the total nitrogen content, 
1 making the amount of graphitic nitrogen in $\# 3$ comparable to that found in $\# 1$ and $\# 2$. These

2 graphitic dopants, in addition to the pyrrolic and pyridinic, explain the inability of sample \#3 to

3 charge properly. It is interesting to note that the Raman spectrum of sample \#3 shows the smallest

$4 \quad D$-band, and therefore the lowest amount of surface defects.

5 To investigate whether the performance could be attributed to defects in the lattice unrelated to 6 nitrogen, a fourth FLG sample that is free of nitrogen was prepared by including a small amount

7 of acetylene during the CVD process in order to induce structural defects throughout the FLG

8 while excluding nitrogen. The presence of defects was confirmed via Raman spectroscopy (Fig.

9 S3). Cathodes made using this FLG were able to reach stage I intercalation at $\sim 2.3 \mathrm{~V}$ as determined 10 by the charging curve of the CV data (Fig. S4), and were able to discharge properly as shown by 11 the peak at $\sim 2.16 \mathrm{~V}$ on the discharge curve. The two peaks found at the lower voltage range of the 12 charge curve were somewhat overlapping, likely due to the presence of internal defects in the FLG 13 which would allow for different stages of intercalation to occur simultaneously. The evolution of 14 chlorine gas was not observed during the charge process as in the case of N-FLG. This result 15 demonstrates that carbon defects, as found in sample \#4, are not as deleterious for intercalation of 16 the $\mathrm{AlCl}_{4}^{-}$ion as nitrogen dopants.

17 In summary, we evaluated N-FLG cathodes based on different methods of imparting nitrogen 18 dopants into the FLG structure. It was found that dopants produced by either in-situ or post-growth 19 doping adversely influence the intercalation of chloroaluminate ions. DFT calculations show that 20 the graphitic type dopants result in the greatest binding energy of the intercalant, thus hindering 21 diffusion through the electrode. The role played by the nitrogen dopants was confirmed by 22 producing nitrogen-free FLG in which defects were induced using acetylene during growth. The 23 electrochemical performance of these defect-engineered FLG samples was similar to pristine FLG, 
1 and were able to achieve full intercalation, demonstrating that the poor performance of the cells is

2 caused by internal nitrogen defects.

\section{Acknowledgments}

4 W. T. and S. E. thank FWO (G.0C60.13N) and the European Union's European Fund for Regional 5 Development and Flanders Innovation \& Entrepreneurship (Accelerate ${ }^{3}$ project, Interreg Vlaanderen6 Nederland program) for financial support. W. T. also thanks the Provincie West-Vlaanderen (Belgium) for 7 his Provincial Chair in Advanced Materials. R. P. and A. M. R thank NASA EPSCoR grant 2022438 and 8 South Carolina EPSCoR stimulus research program 18-SR03. 

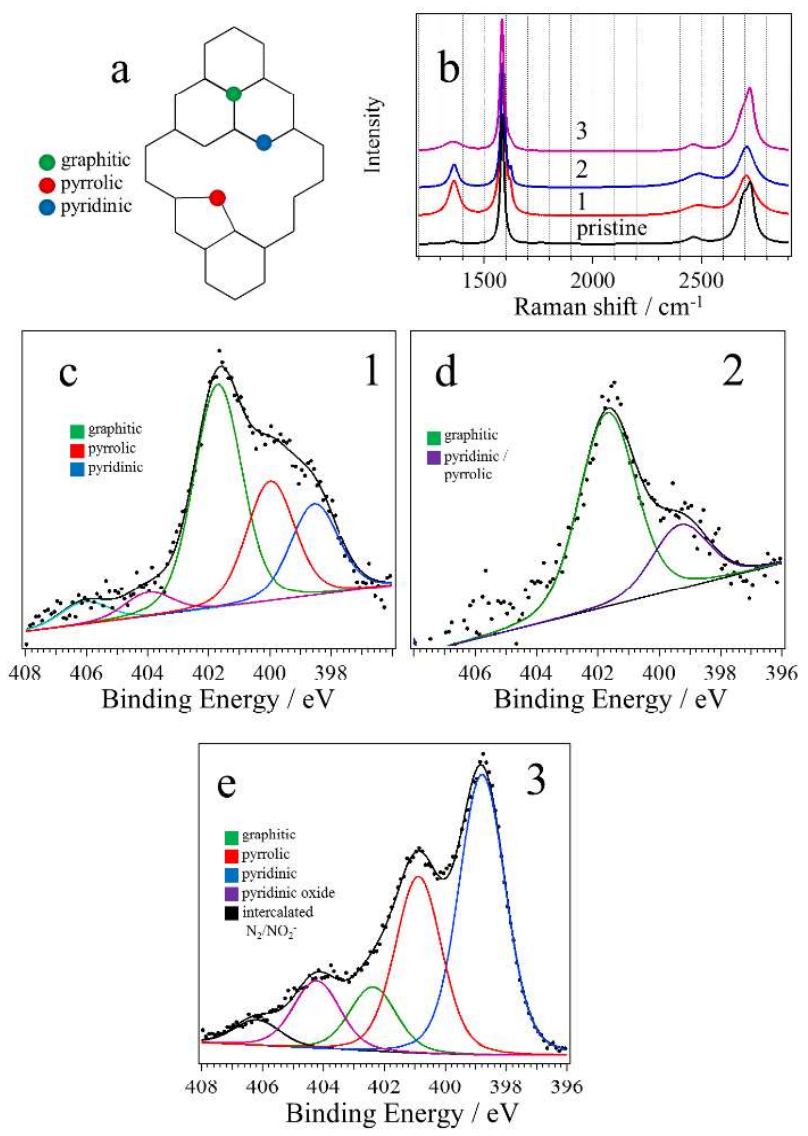

Figure 1. a) Different configurations of nitrogen dopants in the graphene lattice. $b$ ) Raman spectra of N-FLG samples used in this study. c-e) XPS spectra of N-FLG samples which showed samples \#1 and \#2 as predominantly graphitic type, and sample \#3 as the pyridinic type. 

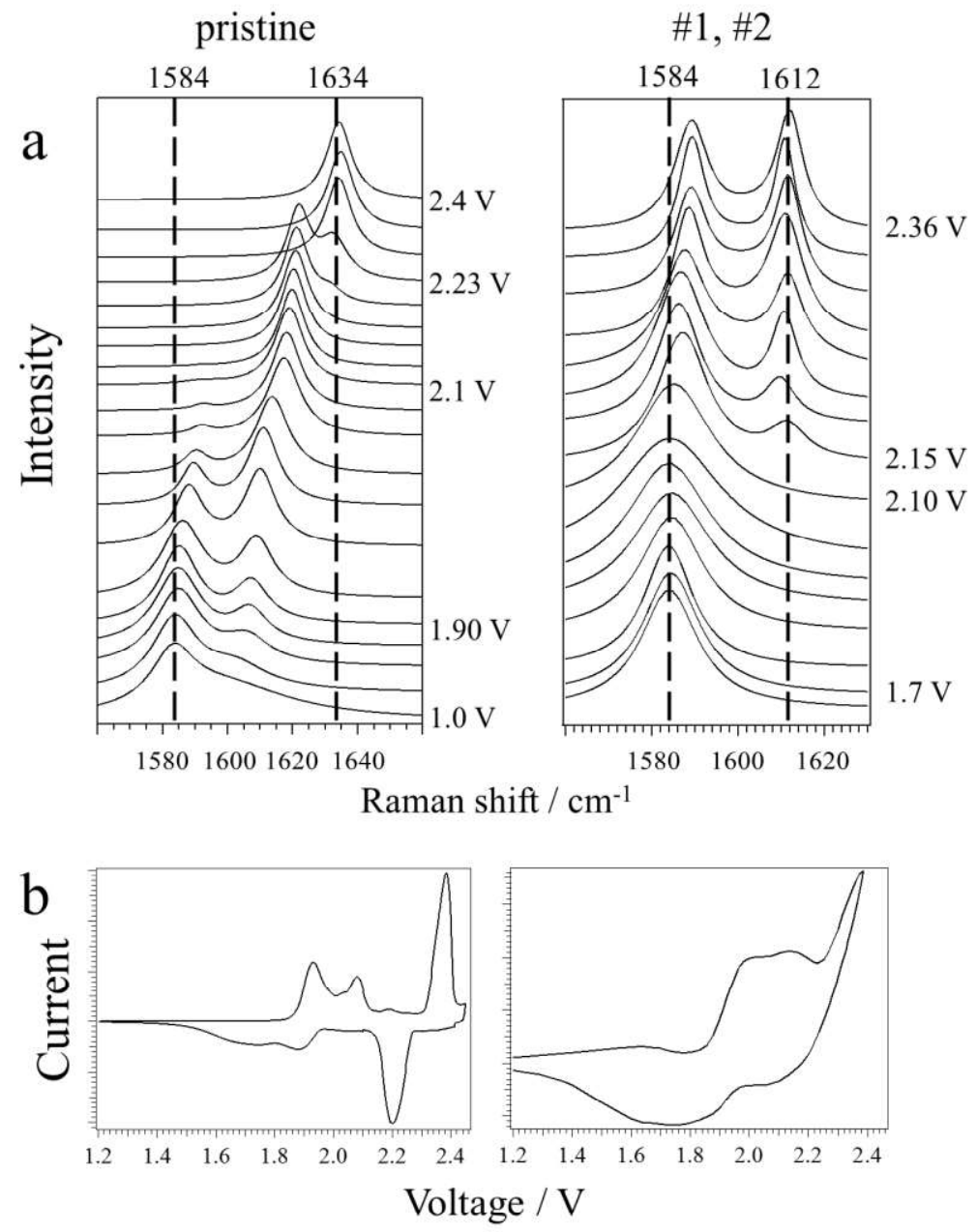

1

Figure 2. The Raman spectra and cyclic voltammograms of samples \#1 and \#2 were very similar, and thus are represented by a single plot for each. a) in situ Raman spectra taken during the charging of pristine and NFLG cathodes. b) Corresponding cyclic voltammograms of these samples. 

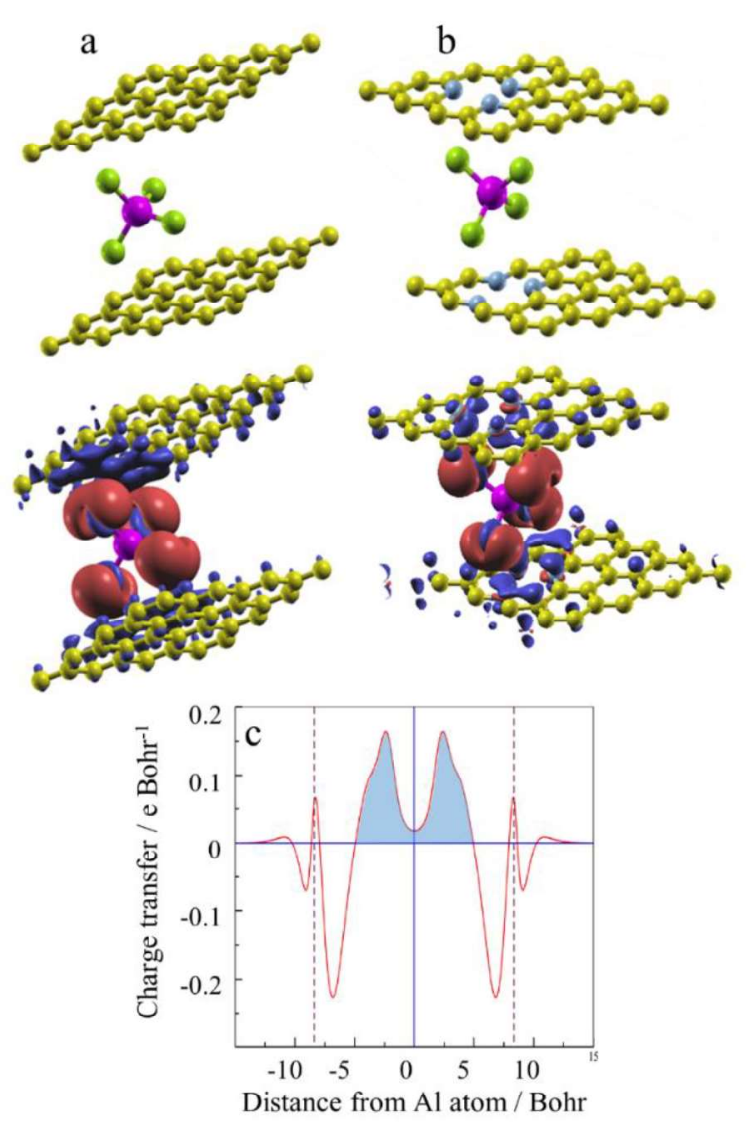

Figure 3. a and b) Models of a pristine and $\mathrm{N}$-doped graphene lattice showing charge transfer to an $\mathrm{AlCl}_{4}^{-}$ion. The pyridinic configuration is shown in this case. c) Charge transfer as a function of distance between the graphene planes and the ion. A similar magnitude of charge transfer was obtained $(\sim 0.8 \mathrm{e})$ for pristine and $\mathrm{N}$-doped samples.

6

References

9 [1] M.C. Lin, M. Gong, B.G. Lu, Y.P. Wu, D.Y. Wang, M.Y. Guan, M. Angell, C.X. Chen, J. 10 Yang, B.J. Hwang, H.J. Dai, An ultrafast rechargeable aluminium-ion battery, Nature. 520 $11 \quad$ (2015) 325. 
[2] J. V. Rani, V. Kanakaiah, T. Dadmal, M.S. Rao, S. Bhavanarushi, Fluorinated Natural Graphite Cathode for Rechargeable Ionic Liquid Based Aluminum-Ion Battery, J. Electrochem. Soc. 160 (2013) A1781-A1784. doi:10.1149/2.072310jes.

[3] S. Wang, Z. Yu, J. Tu, J. Wang, D. Tian, Y. Liu, S. Jiao, A Novel Aluminum-Ion Battery: Al/AlCl 3 -[EMIm]Cl/Ni $3 \mathrm{~S}_{2} @$ Graphene, Adv. Energy Mater. 6 (2016) 1600137. doi:10.1002/aenm.201600137.

[4] X.-G. Sun, Z. Bi, H. Liu, Y. Fang, C.A. Bridges, M.P. Paranthaman, S. Dai, G.M. Brown, A high performance hybrid battery based on aluminum anode and LiFePO 4 cathode, Chem. Commun. 52 (2016) 1713-1716. doi:10.1039/c5cc09019a.

[5] H. Wang, S. Gu, Y. Bai, S. Chen, N. Zhu, C. Wu, F. Wu, Anion-effects on electrochemical properties of ionic liquid electrolytes for rechargeable aluminum batteries, J. Mater. Chem. A. 3 (2015) 22677-22686. doi:10.1039/C5TA06187C.

[6] S.C. Jung, Y.J. Kang, D.J. Yoo, J.W. Choi, Y.K. Han, Flexible Few-Layered Graphene for the Ultrafast Rechargeable Aluminum-Ion Battery, J. Phys. Chem. C. 120 (2016) 1338413389. doi:10.1021/acs.jpcc.6b03657.

[7] L. Zhang, L. Chen, H. Luo, X. Zhou, Z. Liu, Large-Sized Few-Layer Graphene Enables an Ultrafast and Long-Life Aluminum-Ion Battery, Adv. Energy Mater. (2017) 1700034. doi:10.1002/aenm.201700034.

[8] A.S. Childress, P. Parajuli, J. Zhu, R. Podila, A.M. Rao, A Raman spectroscopic study of graphene cathodes in high-performance aluminum ion batteries, Nano Energy. 39 (2017) 69-76. doi:10.1016/j.nanoen.2017.06.038.

[9] Z. Liu, J. Wang, H. Ding, S. Chen, X. Yu, B. Lu, Carbon Nanoscrolls for Aluminum Battery, ACS Nano. 12 (2018) 8456-8466. doi:10.1021/acsnano.8b03961.

[10] X. Yu, B. Wang, D. Gong, Z. Xu, B. Lu, Graphene Nanoribbons on Highly Porous 3D Graphene for High-Capacity and Ultrastable Al-Ion Batteries, Adv. Mater. 29 (2016) 1604118. doi:10.1002/adma.201604118.

[11] K. Share, A.P. Cohn, R. Carter, B. Rogers, C.L. Pint, Role of Nitrogen-Doped Graphene for Improved High-Capacity Potassium Ion Battery Anodes, ACS Nano. 10 (2016) 97389744. doi:10.1021/acsnano.6b05998.

[12] Z. Wang, L. Qie, L. Yuan, W. Zhang, X. Hu, Y. Huang, Functionalized N-doped interconnected carbon nanofibers as an anode material for sodium-ion storage with excellent performance, Carbon N. Y. 55 (2013) 328-334.

doi:10.1016/j.carbon.2012.12.072.

[13] H. Wang, Z. Wu , F. Meng, D. Ma, X. Huang, L. Wang, X. Zhang, Nitrogen-Doped Porous Carbon Nanosheets as Low-Cost, High-Performance Anode Material for SodiumIon Batteries, ChemSusChem. 6 (2013) 56-60. doi:10.1002/cssc.201200680.

[14] A.L.M. Reddy, A. Srivastava, S.R. Gowda, H. Gullapalli, M. Dubey, P.M. Ajayan, Synthesis Of Nitrogen-Doped Graphene Films For Lithium Battery Application, ACS Nano. 4 (2010) 6337-6342. doi:10.1021/nn101926g. 
[15] Y.J. Cho, H.S. Kim, H. Im, Y. Myung, G.B. Jung, C.W. Lee, J. Park, M.-H. Park, J. Cho, H.S. Kang, Nitrogen-Doped Graphitic Layers Deposited on Silicon Nanowires for Efficient Lithium-Ion Battery Anodes, J. Phys. Chem. C. 115 (2011) 9451-9457. doi:10.1021/jp201485j.

[16] H. Wang, C. Zhang, Z. Liu, L. Wang, P. Han, H. Xu, K. Zhang, S. Dong, J. Yao, G. Cui, A.K. Geim, M. Rühle, D.L. Carroll, Nitrogen-doped graphene nanosheets with excellent lithium storage properties, J. Mater. Chem. 21 (2011) 5430. doi:10.1039/c1jm00049g.

[17] N. Ketabi, T. de Boer, M. Karakaya, J. Zhu, R. Podila, A.M. Rao, E.Z. Kurmaev, A. Moewes, T. Hayashi, J. Kong, H. Terrones, G. Dresselhaus, M. Endo, M. Terrones, M.S. Dresselhaus, M.S. Hybertsen, A.N. Pasupathy, Tuning the electronic structure of graphene through nitrogen doping: experiment and theory, RSC Adv. 6 (2016) 56721-56727. doi:10.1039/C6RA07546K.

[18] J. Zhu, A.S. Childress, M. Karakaya, S. Dandeliya, A. Srivastava, Y. Lin, A.M. Rao, R. Podila, Defect-Engineered Graphene for High-Energy- and High-Power-Density Supercapacitor Devices, Adv. Mater. 28 (2016) 7185-7192. doi:10.1002/adma.201602028.

[19] T. Schiros, D. Nordlund, L. Pálová, D. Prezzi, L. Zhao, K.S. Kim, U. Wurstbauer, C. Gutiérrez, D. Delongchamp, C. Jaye, D. Fischer, H. Ogasawara, L.G.M. Pettersson, D.R. Reichman, P. Kim, M.S. Hybertsen, A.N. Pasupathy, Connecting Dopant Bond Type with Electronic Structure in N-Doped Graphene, Nano Lett. 12 (2012) 4025-4031. doi:10.1021/nl301409h.

[20] D. Usachov, A. Fedorov, O. Vilkov, B. Senkovskiy, V.K. Adamchuk, L. V. Yashina, A.A. Volykhov, M. Farjam, N.I. Verbitskiy, A. Grüneis, C. Laubschat, D. V. Vyalikh, The Chemistry of Imperfections in N-Graphene, Nano Lett. 14 (2014) 4982-4988. doi:10.1021/nl501389h.

[21] R. Podila, J. Chacón-Torres, J.T. Spear, T. Pichler, P. Ayala, A.M. Rao, Spectroscopic investigation of nitrogen doped graphene, Appl. Phys. Lett. 101 (2012) 123108. doi:10.1063/1.4752736.

[22] A.C. Ferrari, Raman spectroscopy of graphene and graphite: Disorder, electron-phonon coupling, doping and nonadiabatic effects, Solid State Commun. 143 (2007) 47-57. doi:10.1016/j.ssc.2007.03.052.

[23] L.B. Ebert, Intercalation Compounds of Graphite, Annu. Rev. Mater. Sci. 6 (1976) 181211. doi:10.1146/annurev.ms.06.080176.001145.

[24] M.S. Dresselhaus, G. Dresselhaus, Light scattering in graphite intercalation compounds, in: Solid State Commun., Pergamon Press, 1982: pp. 3-57. doi:10.1007/3540115137_2.

[25] J. Song, D. Chung, P. Ekiund, M. Dresselhaus, Raman Scattering in Graphite Intercalation Compounds, Solid State Commun. 20 (1976) 1111-1115.

[26] J. Wu, L. Ma, R.M. Yadav, Y. Yang, X. Zhang, R. Vajtai, J. Lou, P.M. Ajayan, NitrogenDoped Graphene with Pyridinic Dominance as a Highly Active and Stable Electrocatalyst for Oxygen Reduction, ACS Appl. Mater. Interfaces. 7 (2015) 14763-14769. 
3
[27] Y. Shao, S. Zhang, M.H. Engelhard, G. Li, G. Shao, Y. Wang, J. Liu, I.A. Aksay, Y. Lin, Nitrogen-doped graphene and its electrochemical applications, J. Mater. Chem. 20 (2010) 7491. doi:10.1039/c0jm00782j.

[28] D.S. Su, J. Zhang, B. Frank, A. Thomas, X. Wang, J. Paraknowitsch, R. Schlögl, MetalFree Heterogeneous Catalysis for Sustainable Chemistry, ChemSusChem. 3 (2010) 169180. doi:10.1002/cssc.200900180.

[29] J. Liu, D. Takeshi, D. Orejon, K. Sasaki, S.M. Lyth, Defective Nitrogen-Doped Graphene Foam: A Metal-Free, Non-Precious Electrocatalyst for the Oxygen Reduction Reaction in Acid, J. Electrochem. Soc. 161 (2014) 544-550. doi:10.1149/2.095404jes.

[30] S. Fan, X. Zou, H. Du, L. Gan, C. Xu, W. Lv, Y.-B. He, Q.-H. Yang, F. Kang, J. Li, Theoretical Investigation of the Intercalation Chemistry of Lithium/Sodium Ions in Transition Metal Dichalcogenides, J. Phys. Chem. C. 121 (2017) 13599-13605. doi:10.1021/acs.jpcc.7b05303. 
Supplementary Materials
Click here to download Supplementary Materials: Supplementary information 20190408.docx 